\title{
Combined Orthodontic-surgical Treatment for Skeletal Class III Malocclusion with Multiple Impacted Permanent and Supernumerary Teeth: Case Report
}

\author{
Dai Juan and Feng Xue* \\ Department of Orthodontics, School of Stomatology, Fourth Military Medical University, Xi'an 710032, China
}

\begin{abstract}
In this report we describe a combined orthodontic and surgical treatment for a 14-year-old boy with severe skeletal class III deformity and dental problem. His upper posterior primary teeth in the left side were over-retained and 6 maxillary teeth (bilateral central incisors and canines, left first and second premolars) were impacted, together with 5 supernumerary teeth in both arches. The treatment protocol involved extraction of all the supernumerary and deciduous teeth, surgical exposure and orthodontic traction of the impacted teeth, a bimaxillary orthognathic approach including Lefort I osteotomy. Bilateral sagittal split ramus osteotomy (BSSRO) and genioplasty was performed to correct skeletal problem. After treatment, all of the impacted teeth were brought to proper alignment in the maxillary arch. A satisfied profile and good posterior occlusion was achieved. Treatment mechanics and consideration during different stages are discussed.
\end{abstract}

Keywords: Impacted teeth, orthognathic approach, supernumerary teeth, skeletal class III malocclusion.

\section{INTRODUCTION}

Impaction of multiple permanent teeth is uncommon and few cases have been reported in the literature [1-7]. The causes of eruption disturbances of multiple permanent teeth have been of great interest to the researchers. Both genetic and local environmental factors have been shown to be involved with this phenomenon. A relatively high incidence of multiple impacted teeth often has been detected in patients with craniofacial syndromes (such as cleidocranial dysplasia or Gardner's syndrome) and metabolic disorders [4, 8]. However, in some cases, impaction of multiple teeth is not related to a fixed complex of symptoms [1]. The reasons seem to be supernumerary teeth, insufficient maxillofacial skeletal development and malpositioning of an adjacent tooth and childhood maxillofacial or dentoalveolar trauma [4]. Therefore, patients with multiple impactions cause serious problems in terms of treatment time and outcome and need a thorough clinical and radiographic examination. The participation of a multidisciplinary team including a pediatric dentist, oral surgeon, periodontist, and an orthodontist to accomplish appropriate treatment of such cases is extremely important.

\section{CASE REPORT}

\section{Clinical Examination}

A 14-year-old boy was referred to our department for orthodontic consultation. His chief complaint was upper

\footnotetext{
*Address correspondence to this author at the Department of Orthodontics, School of Stomatology, Fourth Military Medical University, Xi'an, 710032, China; Tel: 86-29-84776137; Fax: 86-29-84776131;
}

E-mail: fengsnow2000@aliyun.com anterior teeth unerupted. His medical history indicated that he used to be weak during childhood and has drug induced deafness. He has normal stature and weight. Intelligence was subjectively normal. No signs of any genetic syndrome and metabolic disorders had been found. He has no reported dental traumatic history. No other family members were known to have similar abnormalities of the teeth and craniofacial skeleton. Clinical and radiographic examination revealed a skeletal Class III malocclusion. Extraorally, he had a relatively concave profile with underdevelopment of the maxilla and excessive mandibular growth. Intraorally, his upper posterior primary teeth in the left side were over-retained and maxillary central incisors, canines and left first premolars were impacted, together with several supernumerary teeth in both arches. Slight anterior crowding was noted in lower arch (Fig. 1). Cephalometric analysis indicated features of skeletal class III. The maxilla was retrusive $\left(\mathrm{SNA}=75^{\circ}\right.$ ), while the mandible was normal $\left(\mathrm{SNB}=78^{\circ}\right)$ in central occlusion, indicating a skeletal Class III $\left(\mathrm{ANB}=-3^{\circ}\right)$. The mandible showed a forward and downward rotation and a hyperdivergent skeletal pattern $\left(\mathrm{FMA}=36^{\circ}, \mathrm{SN}-\mathrm{GoGn}=39^{\circ}\right)$. He denied ever having any temporomandibular joint (TMJ) dysfunction signs or symptoms, and maximal opening and lateral and anterior movements were within normal limits.

\section{Treatment Planning}

1. Extraction of all the supernumerary teeth.

2. Aligning and leveling upper teeth and open space for the impacted teeth.

3. Extraction of both upper lateral incisors.

4. Surgical exposure and orthodontic traction of the impacted teeth individually. 

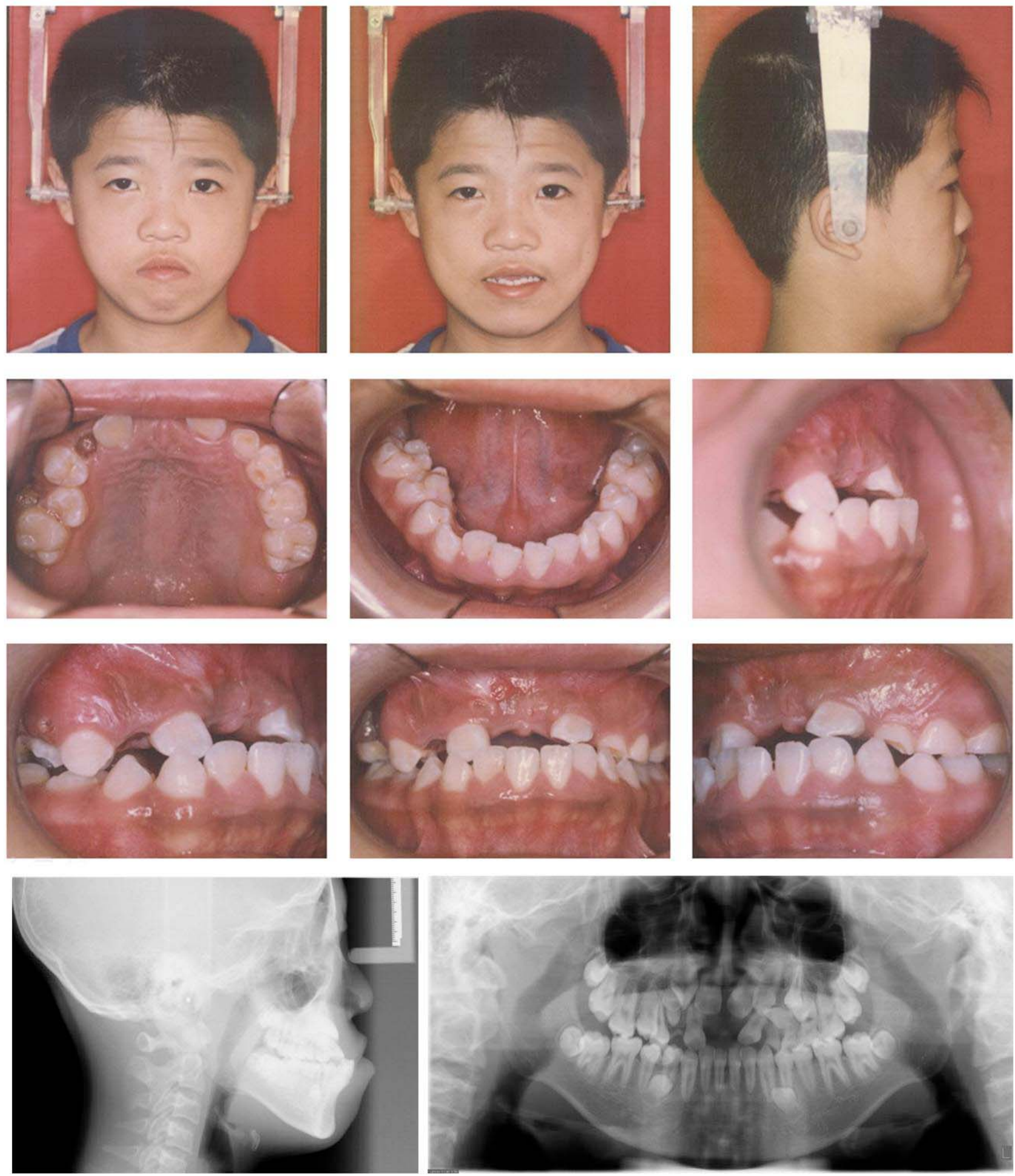

Fig. (1). Pre-treatment facial, intraoral photos and radiographs.

5. Maxillary Lefort I osteotomy, BSSRO and genioplasty to improve skeletal maxillary retrusion, mandibular protrusion and chin position.

6. Post-surgical orthodontic treatment to establish the ideal occlusal relationship with MEAW technology.

7. Retention to maintain proper occlusion after treatment.

\section{Treatment Progress}

After extraction of all the supernumerary and lower deciduous teeth, the $0.022 \times 0.028$ in slot straight-wire fixed appliance was used to level maxillary and mandible teeth. Once the upper teeth were aligned (for 6 months with 0.012, 0.014 and 0.016 NITI wire) and space recovered for the impacted central incisors by means of the nitinol spring (for 6 months with $0.5 \mathrm{~mm}$ stainless steal wire), surgery assisted 

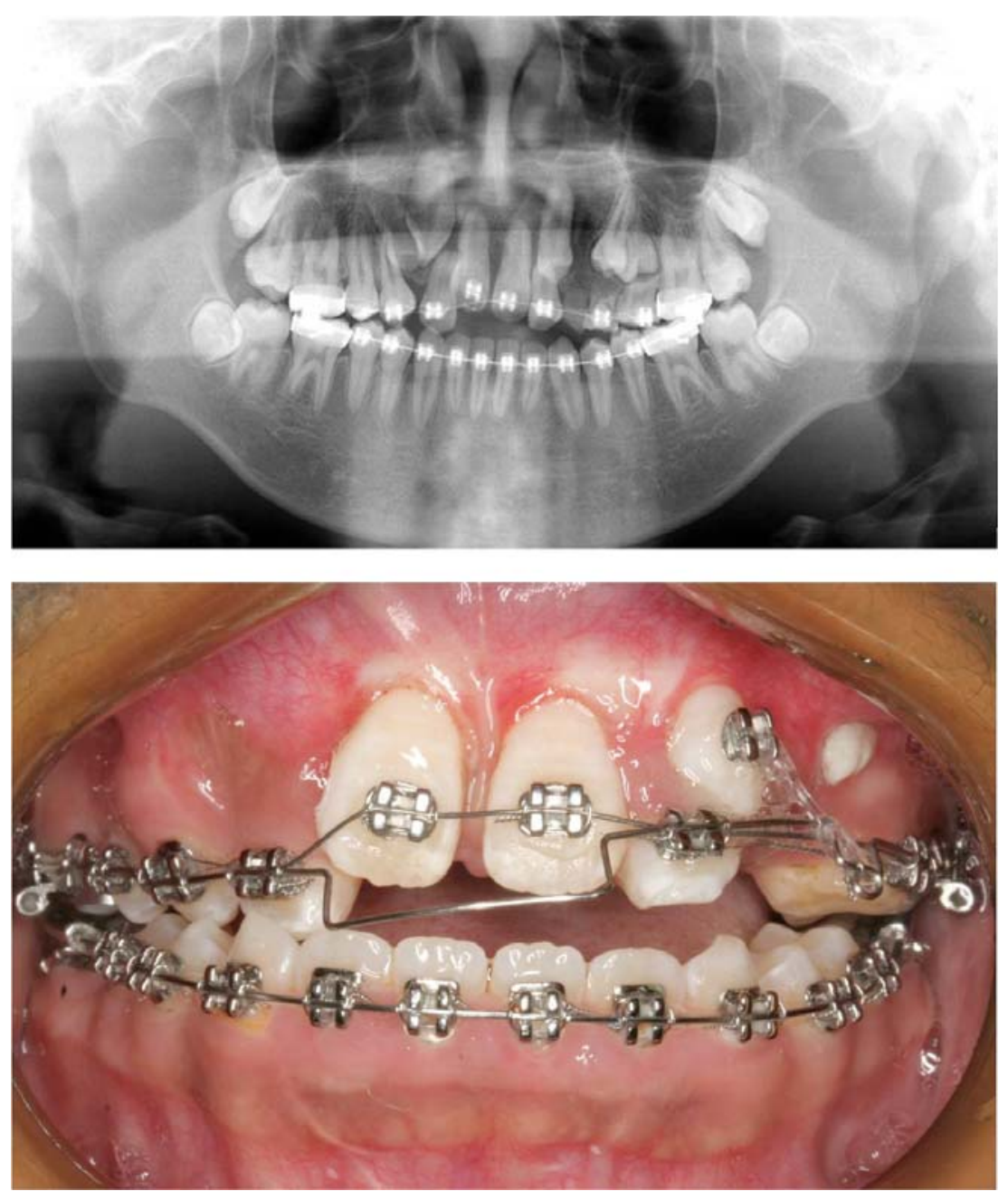

Fig. (2). Mid-treatment intraoral photo and radiograph.

traction for central incisor was performed individually. In brief, the labial surface of the maxillary central incisor was surgically exposed. An attachment was then bonded to the teeth surface and the flap fully sutured back to the former position. The twisted stainless steel ligature wire $(0.2 \mathrm{~mm})$ that has been previously placed in the attachment was then drawn occlusally with the main archwire of $0.5 \mathrm{~mm}$ stainless steel wire. Ten months later, both central incisors erupted into the oral cavity, the brackets were banded and proper guidance was provided to the central incisors during normal eruption. In order to create space for the impacted upper canines and premolars, bilateral upper lateral incisors with severe root resorption and retained primary teeth were extracted. Then, surgery assisted eruption guidence for impacted canines and premolars were performed individually. The main archwire of $0.5 \mathrm{~mm}$ stainless steel wire with loop was placed, the power chain was connected between impacted canine and the loop. Four months later, the canine was guided into the upper arch (Fig. 2). After 10 months of alignment of all the dentition (with $0.016,0.016 \times 0.022$, $0.017 \times 0.025,0.018 \times 0.025$ NITI wire), $.018 \times .025$ stainless steel wire was placed. In this stage, all the impacted teeth were guided to normal position, the patient and parents were satisfied with the teeth but disliked the concave face (Fig. 3). Hence, the patient was suggested to receive surgery. The bimaxillary orthognathic approach including Lefort I osteotomy. Bilateral sagittal split ramus osteotomy (BSSRO) and genioplasty was performed to correct skeletal problem. Titanium screws were used for rigid fixation. Maxillomandibular fixation with an occlusal splint was performed for 4 weeks. The MEAW technology $(0.018 \times 0.025$ stainless steel wire $)$ together with vertical elastic and short class III elastic was performed to establish the ideal occlusal relationship. After 8 months of post-surgical orthodontic treatment, Ideal occlusion was achieved and the fixed appliance was removed. Clear retainers were worn in both jaws. The retainers were maintained all day for 6 months, subsequently, were worn only during the night for another 1 year. Total active treatment period lasted for 5 years including 1 year missing for operation fee preparation.

\section{Treatment Results}

After combined orthodontic-surgical treatment, all treatment objectives were fully achieved including an ideal overjet and overbite with well-interdigitated class II molar relationship. The repositioned upper bilateral canines and first premolars moved mesially and took the physiological positions of lateral incisors and canines individually with slight occlusal adjustment. The incisors and canines had an 

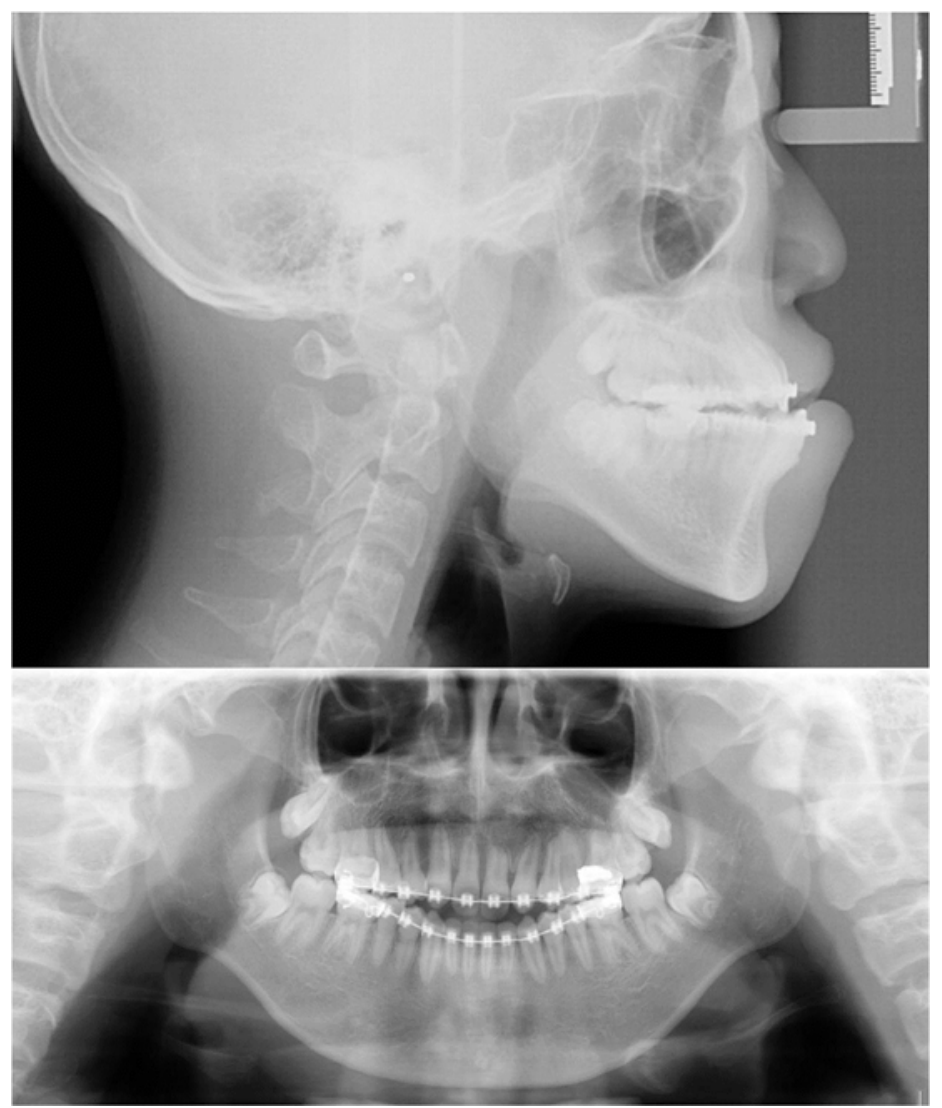

Fig. (3). Presurgical radiograph.
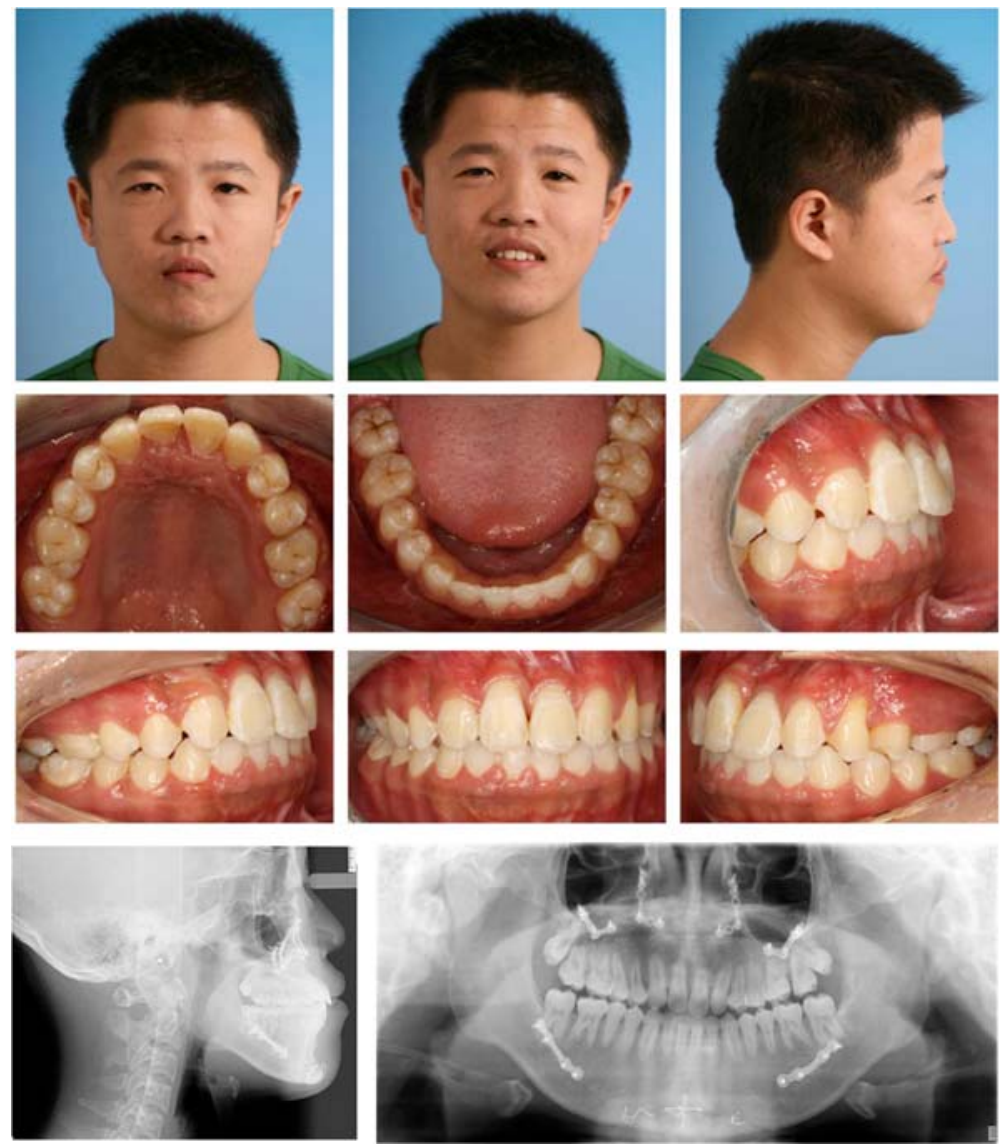

Fig. (4). Post-treatment facial, intraoral photos and radiographs. 
Table 1. Cephalometric data.

\begin{tabular}{|c|c|c|c|}
\hline Measurements & Pretreatment & Posttreatment & Norm \\
\hline \hline SNA & 75 & 82 & $82.8 \pm 4.0$ \\
\hline SNB & 78 & 2 & $80.1 \pm 3.9$ \\
\hline ANB & -3 & 33 & $2.7 \pm 2.0$ \\
\hline U1 to NA plane ( $\left.{ }^{\circ}\right)$ & 39 & 9.5 & $5.1 \pm 2.4$ \\
\hline U1 to NA plane (mm) & 11 & 28 & $30.3 \pm 5.8$ \\
\hline L1 to NB plane ( $\left.{ }^{\circ}\right)$ & 29 & 1 & $6.7 \pm 2.1$ \\
\hline L1 to NB plane (mm) & 1.5 & 119 & $124.2 \pm 8.2$ \\
\hline Interincisal angle & 111 & 38 & $31.1 \pm 5.6$ \\
\hline SN-GoGn & 39 & 36 & $31.3 \pm 5.0$ \\
\hline FMA & 36 & 88 & $93.9 \pm 6.2$ \\
\hline IMPA & 90.5 & 53.5 & 56 \\
\hline FMIA & & $54.9 \pm 6.1$ & \\
\hline
\end{tabular}

acceptable gingival contour. The post-treatment radiograph revealed no periodontal bone loss, minimal root resorption, acceptable root parallelism of the newly positioned incisors and canines (Fig. 4). The cephalometric data showed that the sagital skeletal relationship was corrected from Class III to normal and the mandiblular plane was maintained (Table 1).

\section{DISCUSSION}

Eruption failures of multiple teeth can cause serious dental and aesthetic difficulties as well as psychological problems. The impaction of multiple teeth occurs infrequently, the etiologic factors differ, including genetic syndrome, metabolic disorders and various local causes. The arrested eruption of multiple teeth in the case presented here, are probably caused by the presence of multiple supernumerary teeth and insufficient maxillofacial skeletal development. The reported incidence of supernumerary teeth is usually higher in males than in females. It ranges from $0.5 \%$ to $5.3 \%$ in the permanent dentition [9]. In most cases, multiple supernumerary teeth are associated with various syndromes, however, some cases also could not detect any associated systemic conditions or syndromes [10]. Hence, the exact etiology of multiple impacted supernumerary and permanent teeth in this case remain unclear and requires further investigation.

Management for the impaction of multiple permanent teeth is a challenge to the orthodontists. Before treatment, the impacted teeth require an accurate diagnosis and localization. The position of the impacted tooth, the degree of root development, the degree of dilacerations, the adjacent teeth, and the presence of space should be carefully considered to determine whether successful alignment of the impacted tooth can be achieved. For this case, the combination of conventional radiographic techniques including panoramic, and lateral cephalometric radiographs was used to evaluate the labiolingual position, root development and the adjacent structures. However, the two-dimensional radiographs can sometimes unjustifiably suggest a bad prognosis of an impacted tooth. Cone-beam CT scanning, which gives a three-dimensional image, can provide important additional diagnostic information [11, 12]. A combination of surgical exposure and orthodontic traction is an optimum to deal with multiple impactions. Surgical transplanting may be another choice, however, surgical transplanting or repositioning of teeth generally has a high risk of complications, such as ankylosis, pulp necrosis, and root resorption [13].

As to this patient, the surgical-orthodontic treatment of multiple impacted teeth yielded good esthetic and periodontal results. Firstly, the surgical traction on the bilateral central incisors were performed individually, the maxillary deciduous first and second molars in the left side were maintained in the first leveling of the arches and then as anchorage. After the eruption of the central incisors, no enough space for aligning other impacted teeth, considering the existing resorbed root of upper lateral incisors, extraction of bilateral upper lateral incisors was performed allowing for the eruption of impacted canines. The palatal arch was used to increase the anchorage only after the extraction of the second deciduous molar. The erupted upper left second premolar was in a $180^{\circ}$ buccal-lingual rotation position and no enough room for correcting this rotation. So just left it. The lingual cusp of this tooth was elongated for buccal alignment. The occlusal adjustment was made on the buccal cusp.

After all the impacted teeth were guided tonormal position, the patient and parents were satisfied with the aligned teeth but disliked the concave face. Regarding his deficient upper lip and maxilla, proclined mandible and retroclined chin, a single jaw surgery could not solve all the problems. Hence, a bimaxillary orthognathic approach was performed, including Lefort I osteotomy for moving maxilla forward. Bilateral sagittal split ramus osteotomy (BSSRO) for moving 
mandible backward and genioplasty for moving chin forward and upward.

\section{CONCLUSION}

To achieve optimum aesthetic and functional results, a multidisciplinary cooperation between the oral surgeon, orthodontist, peridontist, prosthodontist and pedodontist should be performed for the effective management of such cases. The protocol of comprehensive treatment of multiple impaction contains serial removal of primary teeth and supernumerary teeth, assisting the eruption of the permanent teeth by surgical exposure and orthodontic traction and facial improvement by orthognathic surgery. Any impacted tooth which couldn't be guided in the normal position after surgical or orthodontic methods requires extraction and replacement. Furthermore, long-term stability and periodontal health of the impacted teeth should be monitored routinely.

\section{PATIENT'S CONSENT}

Available on website.

\section{CONFLICT OF INTEREST}

The authors confirm that this article content has no conflicts of interest.

\section{ACKNOWLEDGEMENTS}

Declared none.

\section{REFERENCES}

[1] Zuccati GC, Doldo T. Multiple bilateral impactions in an adolescent girl. Am J Orthod Dentofacial Orthop 2010; 137 (Suppl 4): S163-72.

[2] Kulkarni VK, Reddy S, Duddu M, et al. Multidisciplinary management of multiple maxillary anterior supernumerary teeth: a case report. Quintessence Int 2010; 41(3): 191-5.

[3] Tanaka E, Kawazoe A, Nakamura S, et al. An adolescent patient with multiple impacted teeth. Angle Orthod 2008; 78(6): 1110-8.

[4] Bayar GR, Ortakoglu K, Sencimen M. Multiple impacted teeth: report of 3 cases. Eur J Dent 2008; 2(1): 73-8.

[5] Conley RS, Boyd SB, Legan HL, et al. Treatment of a patient with multiple impacted teeth. Angle Orthod 2007; 77(4): 735-41.

[6] Yildirim D, Yilmaz HH, Aydin U. Multiple impacted permanent and deciduous teeth. Dentomaxillofac Radiol 2004; 33(2): 133-5.

[7] Babu V, Nagesh KS, Diwakar NR. A rare case of hereditary multiple impacted normal and supernumerary teeth. J Clin Pediatr Dent 1998; 23(1): 59-61.

[8] Mohan RP, Suma GN, Vashishth S, et al. Cleidocranial dysplasia: clinico-radiological illustration of a rare case. J Oral Sci 2010; 52(1):161-6.

[9] Wang XP, Fan J. Molecular genetics of supernumerary tooth formation. Genesis 2011; 49(4): 261-77.

[10] Inchingolo F, Tatullo M, Abenavoli FM, et al. Non-syndromic multiple supernumerary teeth in a family unit with a normal karyotype: case report. Int J Med Sci 2011; 7: 378-84

[11] Dalessandri D, Laffranchi L, Tonni I, et al. Advantages of cone beam computed tomography (CBCT) in the orthodontic treatment planning of cleidocranial dysplasia patients: a case report. Head Face Med 2011; 27: 7-6.

[12] Wang WM, Wang X, Wang TM. Evaluation of nonsyndromic multiple supernumerary teeth using three-dimensional computerized tomography: a case report and literature review. J Contemp Dent Pract 2010; 11(1): E081-7.

[13] McAboy, CP, Grumet JT, Siegel EB, et al. Surgical uprighting and repositioning of severely impacted mandibular second molars. J Am Dent Assoc 2003; 134:1459-62.

Received: February 01, 2014

Revised: April 04, 2014

Accepted: April 12, 2014

(C) Dai and Feng; Licensee Bentham Open.

This is an open access article licensed under the terms of the Creative Commons Attribution Non-Commercial License (http://creativecommons.org/licenses/by-nc/3.0/) which permits unrestricted, non-commercial use, distribution and reproduction in any medium, provided the work is properly cited. 